\title{
Pulmonary puzzle: A rare case of dysphagia
}

S Sinha Roy, MBBS, DTCD, MD, FCCP; C F N Koegelenberg, MB ChB, MMed (Int), FCP (SA), FRCP (UK), Cert Pulm (SA), PhD; E M Irusen, MB ChB, FCP (SA), FCCP, PhD

Division of Pulmonology, Department of Medicine, Stellenbosch University and Tygerberg Hospital, Cape Town, South Africa

Corresponding author: S Sinha Roy (sinharoy@gmail.com)

S Afr Respir J 2016;22(3):73-75. DOI:10.7196/SARJ.2016.v22i3.81

\section{Case report}

A 58-year-old man presented with a longstanding history of dysphagia and sensation of something lodged in the back of his throat. He had no significant past medical history, was a smoker (30 pack-years) and used $>60$ units of alcohol per week. A physical examination was unremarkable. The patient was referred to the respiratory clinic at Tygerberg Hospital, Cape Town, by a specialist physician who was concerned about the patient's abnormal chest radiograph (Fig. 1).

The chest radiograph showed that the normal left-sided aortic arch and the usual companion descending aorta were absent. The arch coursed to the right with the descending aorta also on the right. A computed tomography (CT) scan (Figs 2 and 3) and reconstructions (Figs 4 and 5) confirmed the right-sided aortic arch with a retrooesophageal aberrant left subclavian artery and formation of a Kommerell's diverticulum at the origin. In the absence of major complications, he was managed conservatively.

\section{Discussion}

The prevalence of a right-sided aortic arch among adults is $\sim 1 / 1000$ 2000 of the population. ${ }^{[1]}$ With this rare congenital anomaly, the aortic arch and the descending thoracic aorta are situated in the right hemithorax. During the third week of gestation, the aortic arch

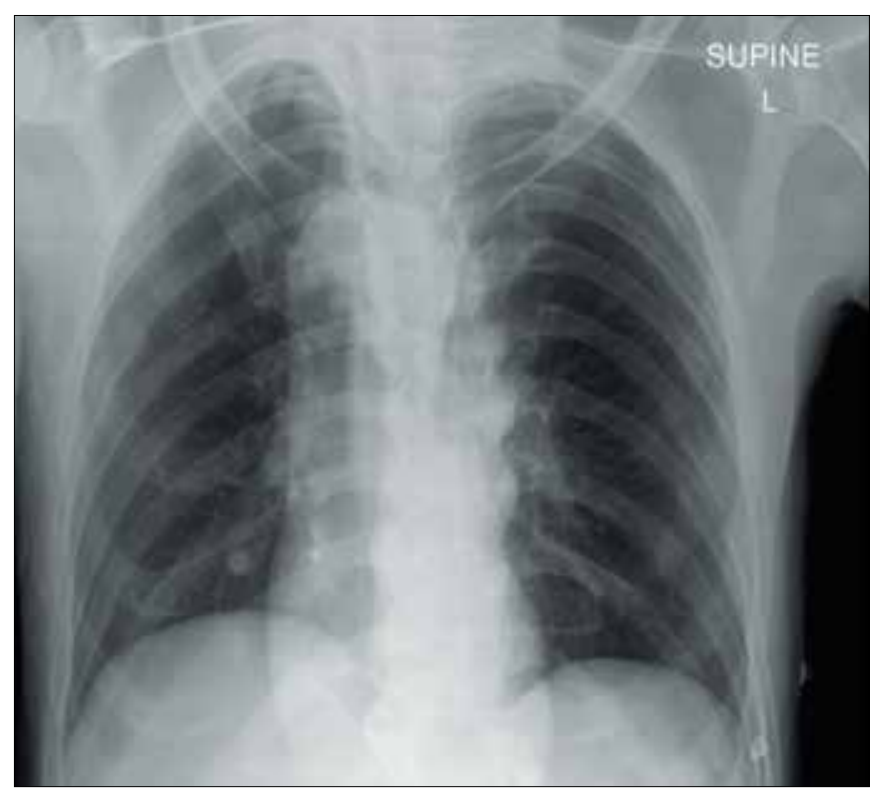

Fig. 1. The patient's chest radiograph that prompted the referral. develops. The arch is formed by the left fourth aortic arch vessel while the right fourth vessel regresses. ${ }^{[1]}$ The two dorsal aortas fuse (or the right dorsal aorta involutes) to form the descending aorta, and the ventral aortic limbs fuse to form the aortic sac. In individuals with a right-sided aortic arch, the right dorsal aorta and right fourth

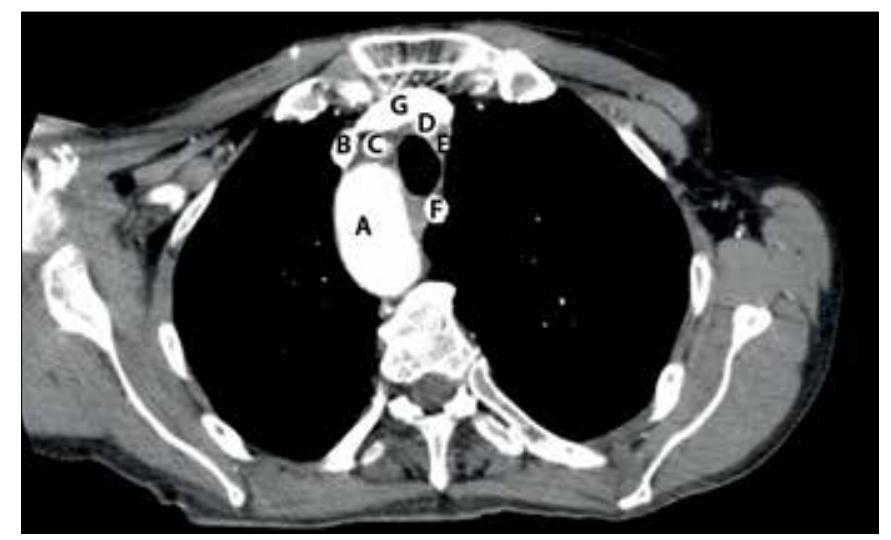

Fig. 2. Contrasted CT thorax mediastinal view showing a right-sided aortic arch with its branches: (A) the aortic arch (right-sided); (B) superior vena cava; $(C)$ right subclavian artery; $(D)$ right common carotid artery; (E) left common carotid artery; (F) aberrant left subclavian artery; and $(G)$ left brachiocephalic vein draining into SVC.

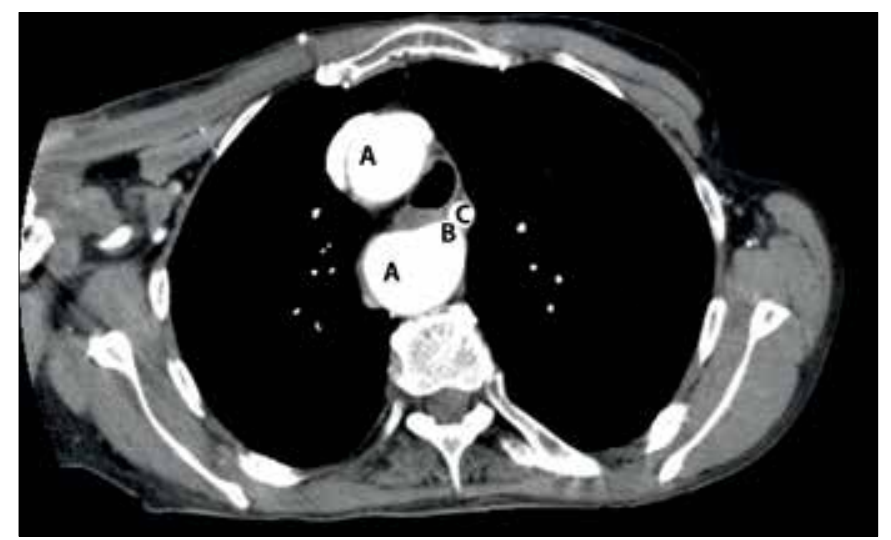

Fig. 3. Contrasted CT scan of the patient's thorax, mediastinal view, showing aberrant left subclavian artery with formation of a Kommerell's diverticulum: (A) the aortic arch (right-sided) indenting on the trachea; (B) Kommerell's diverticulum; and $(C)$ aberrant left subclavian artery forming the diverticulum. 
branchial arch persist and the distal left dorsal aorta disappears. ${ }^{[1]}$ These variants may have occasional complications, mostly tracheobronchial or oesophageal compression and, rarely, disturbances of normal blood flow patterns, ${ }^{[2]}$ e.g. cerebral ischaemia because of reversal of flow through the vertebrobasilar vasculature. Several types have been described ${ }^{[1,3]}$ (Fig. 6). Type I (15 - 20\% of cases) involves a rightsided aortic arch with mirror-image branching. This type is usually associated with congenital heart disease, the most common being tetralogy of Fallot or pulmonary stenosis. Type II is the most common variety (around $80 \%$ of all cases), in which a right-sided aortic arch is present with an aberrant left subclavian artery. The vessels originate in the following order: left common carotid, right common carotid, right



Fig. 4. CT scan of patient's thorax, reconstructed view showing right arch and its branches. subclavian and left subclavian artery. A Kommerell's diverticulum develops at the junction of the aberrant left subclavian artery with the right aortic arch and may cause compressive features ${ }^{[3,4]}$ such as dysphagia, dyspnoea, stridor, wheezing, cough, choking spells or chest pain. The dysphagia caused by the aberrant left subclavian artery causing a vascular ring formation is sometimes referred to as dysphagia pseudolusoria. ${ }^{[1]}$ The type III malformation is very rare: a right-sided aortic arch is present with isolation of the left subclavian artery. The left subclavian artery does not arise from the arch or

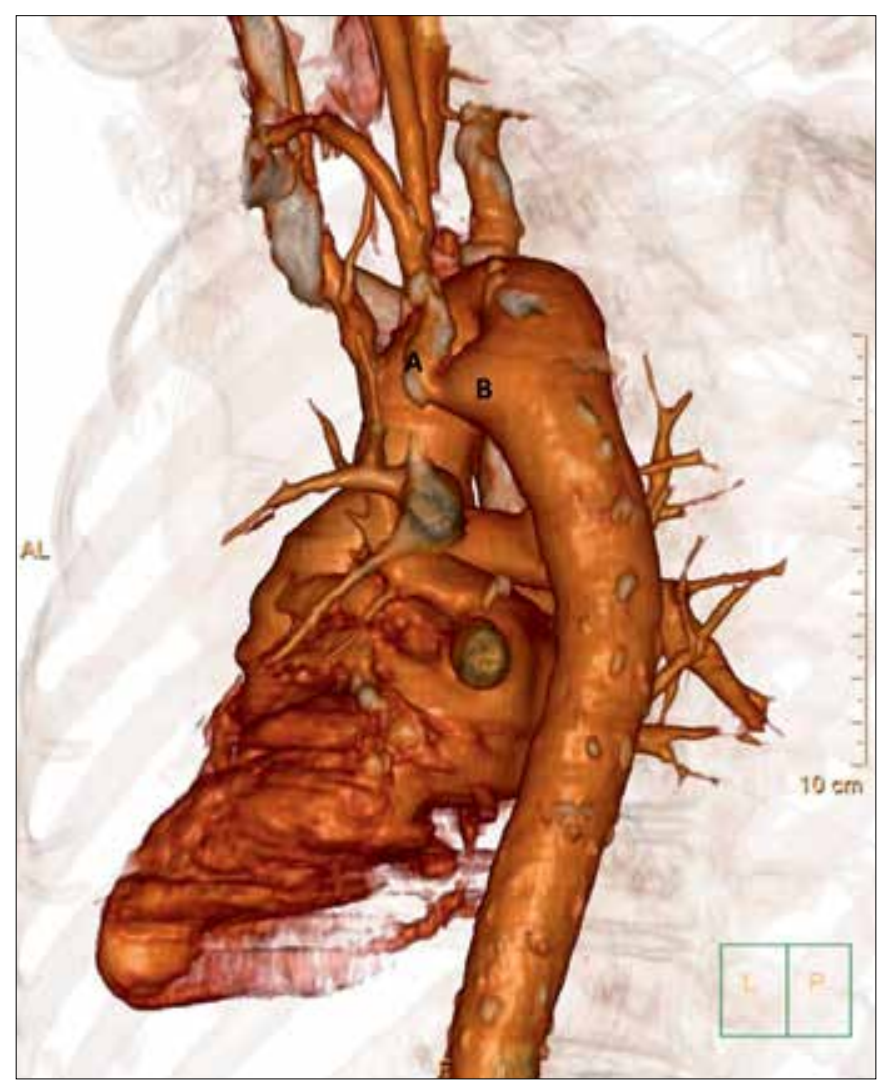

Fig. 5. CT scan of thorax of patient, reconstructed view showing aberrant left subclavian artery originating from right arch from its posteromedial aspect with the formation of Kommerell's diverticulum: $(A)$ the aberrant left subclavian artery and (B) Kommerell's diverticulum. Note the origin of the aberrant left subclavian artery below the right subclavian artery.

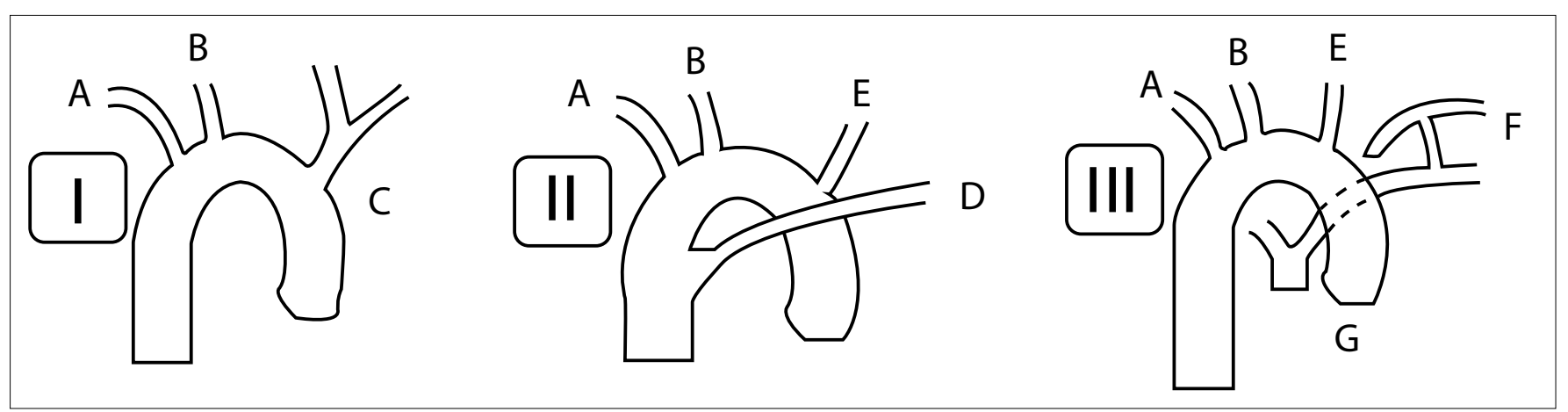

Fig. 6. The three main variations seen in patients with right-sided aortas: type I, mirror image aortic arch; type II, aberrant left subclavian artery; type III, isolated subclavian artery. (A) the right subclavian artery; (B) right common carotid artery; (C) brachiocephalic trunk; (D) aberrant left subclavian artery; (E) left common carotid artery; (F) ductus arteriosus; and $(G)$ pulmonary trunk. 
the descending aorta. It may be associated with vertebrobasilar insufficiency and subclavian steal syndrome. This variety may also be associated with congenital heart disease.

The causes of right-sided aortic arch are unknown. In some patients, 22q11 deletions have been found. ${ }^{[5]}$ This deletion is responsible for the DiGeorge, velocardiofacial and conotruncal face anomaly syndromes, which are often referred to by the unified terms CATCH-22 syndrome or chromosome 22q11 deletion syndrome.

A right-sided aortic arch alone is usually asymptomatic and diagnosed incidentally. During pregnancy, a prenatal ultrasound may reveal the abnormal course of the arch. Chest radiography demonstrates an absent left aortic knuckle and left-sided descending aorta. The right-sided aortic arch is discernible, often at a relatively higher plane, and may project like a mass in the immediate right lower paratracheal region (this, in fact, represents the most common misdiagnosis). The course of the right-sided descending aorta can then be traced. The lower trachea may be pushed to the left or indented by the right arch. Rarely, the arch may be associated with aneurysmal dilatation and other anomalies of position and branching of the aorta. A CT scan of the thorax will delineate the exact anatomical abnormality. Magnetic resonance angiography and a barium swallow test may be considered, to better demonstrate the abnormalities and vascular anomalies.

\section{References}

1. Kau T, Sinzig M, Gasser J, et al. Aortic development and anomalies. Semin Intervent Radiol 2007;24(2):141-152. DOI:10.1055/s-2007-980040

2. Białowas J, Hreczecha J, Grzybiak M. Right-sided aortic arch. Folia Morphol 2000;59(3):211-216.

3. Cina CS, Arena GO, Bruin G, Clase CM. Kommerell's diverticulum and aneurysmal right-sided aortic arch: A case report and review of the literature. J Vasc Surg 2000;32(6):1208-1214. DOI:10.1067/mva.2000.108012

4. Salanitri J. MR angiography of aberrant left subclavian artery arising from right-sided thoracic aortic arch. Br J Radiol 2005;78(934):961-966. DOI:10.1259/bjr/20210494

5. McElhinney DB, McDonald-McGinn D, Zackai EH, Goldmuntz E. Cardiovascular anomalies in patients diagnosed with a chromosome 22q11 deletion beyond 6 months of age. Pediatrics 2001;108(6):E104. DOI:10.1542/peds.108.6.e104 